\title{
Iridocorneal Endothelial Syndrome with Coexisting Macular Edema and Neurosensory Detachment: An Unusual Case Report
}

\author{
Suneeta Dubey ${ }^{1}$, Kanika Jain ${ }^{2}$, Shalini Singh ${ }^{3}$, Saptarishi Mukhejee ${ }^{4}$
}

\begin{abstract}
The iridocorneal endothelial (ICE) syndrome is a sporadic, unilateral condition characterized by abnormalities of the cornea, anterior chamber angle, and iris affecting middle-aged women (3rd to 5th decade). It consists of three variants: progressive iris atrophy, Cogan-Reese syndromediffuse nevus or iris nodules, and Chandler syndrome-corneal endothelial abnormalities leading to corneal edema. This syndrome has an abnormal corneal endothelial cell layer (proliferative endotheliopathy), which migrates across the angle and onto the surface of the iris. It is a potentially blinding condition as a result of secondary glaucoma and/or corneal decompensation. Although few case reports have shown an association of ICE syndrome with macular edema; however, our case was unique in the sense that it was associated with neurosensory detachment (NSD). It can be suggested that PG analogs are not a good idea to be prescribed for secondary glaucoma management in patients with ICE syndrome as it can predispose to the development of macular edema with NSD.

Keywords: Cogan-Reese syndrome, ICE syndrome, Iridocorneal endothelial syndrome, Macular edema, Neurosensory detachment, Progressive iris atrophy.

Journal of Current Glaucoma Practice (2021): 10.5005/jp-journals-10078-1315
\end{abstract}

\section{INTRODUCTION}

The iridocorneal endothelial (ICE) syndrome is typically a sporadic, unilateral condition characterized by abnormalities of the cornea, anterior chamber angle, and iris affecting middle-aged women ( 3 rd to 5 th decade). It consists of three very rare and frequently overlapping disorders according to the appearance of iris and cornea: progressive iris atrophy characterized by severe iris changes, Cogan-Reese syndrome characterized by diffuse nevus or iris nodules, and Chandler syndrome characterized by corneal endothelial abnormalities leading to corneal edema. In clinical practice, the progressive course of the disease often leads to a challenging diagnosis with a large percentage of cases appearing as mixed forms in the different stages of the disease. This syndrome has an abnormal corneal endothelial cell layer (proliferative endotheliopathy), which migrates across the angle and onto the surface of the iris. Secondary angle-closure glaucoma occurs in ICE syndrome due to contraction of abnormal tissue in the angle. It is a potentially blinding condition as a result of secondary glaucoma and/or corneal decompensation. ${ }^{1-4}$ Although few case reports have shown an association of ICE syndrome with macular edema, ${ }^{5-7}$ none of these had associated neurosensory detachment (NSD) as was seen in our patient.

\section{Case Description}

A 35-year-old woman with no known systemic ailment, resident of Delhi, had complaints of unilateral left-sided episodes of headache, whereby she was diagnosed as having left eye ICE syndrome elsewhere 6 months back. On presentation in January 2018, she was on maximum medical therapy in the form of eye drop brimonidine $0.1 \% \mathrm{BD}$, timolol $0.5 \% \mathrm{BD}$, dorzolamide $0.2 \% \mathrm{BD}$, and travoprost $0.004 \%$ HS. Medical history (prolonged corticosteroid use, hypertension, and smoking) and family history were unremarkable.
1,4 Department of Glaucoma, Dr Shroff's Charity Eye Hospital, New Delhi, India

2Department of Ophthalmology, Deen Dayal Upadhyay Hospital, Hari Nagar, Delhi, India

${ }^{3}$ Department of Ophthalmology, Dr Shroff's Charity Eye Hospital, New Delhi, India

Corresponding Author: Kanika Jain, Department of Ophthalmology, Deen Dayal Upadhyay Hospital, Hari Nagar, Delhi, India, Phone: +91 9818018301, e-mail: kanikajain024@gmail.com

How to cite this article: Dubey S, Jain K, Singh S, et al. Iridocorneal Endothelial Syndrome with Coexisting Macular Edema and Neurosensory Detachment: An Unusual Case Report. J Curr Glaucoma Pract 2021;15(3):149-152.

Source of support: Nil

Conflict of interest: None

She was not having a type A personality and she was not pregnant. A systemic evaluation was within normal limits.

On ocular examination, the best-corrected visual acuity (BCVA) was $6 / 6$ in both eyes. A slit-lamp evaluation was within normal limits in the right eye whereas, in the left eye, she had clear cornea, multiple iris holes (at 4, 5, 9 o'clock), corectopia, a vertically oval pupil with a lost iris pattern. Peripheral anterior synechiae (PAS) were present at the 2-3 o'clock position and the anterior chamber had variable depth in the left eye (Fig. 1). Intraocular pressure (IOP) as measured by Goldmann applanation tonometry (GAT) was 12 and $20 \mathrm{~mm} \mathrm{Hg}$ in right and left eye, respectively, and diurnal variation in the left eye showed unstable maintenance of IOP even on maximal medical therapy. Gonioscopy showed open angles in the right eye and closed-angle with interspersed broad-based PAS in all quadrants in the left eye (Fig. 2). Disk showed a cup disk ratio

(c) The Author(s). 2021 Open Access This article is distributed under the terms of the Creative Commons Attribution 4.0 International License (https://creativecommons. org/licenses/by-nc/4.0/), which permits unrestricted use, distribution, and non-commercial reproduction in any medium, provided you give appropriate credit to the original author(s) and the source, provide a link to the Creative Commons license, and indicate if changes were made. The Creative Commons Public Domain Dedication waiver (http://creativecommons.org/publicdomain/zero/1.0/) applies to the data made available in this article, unless otherwise stated. 

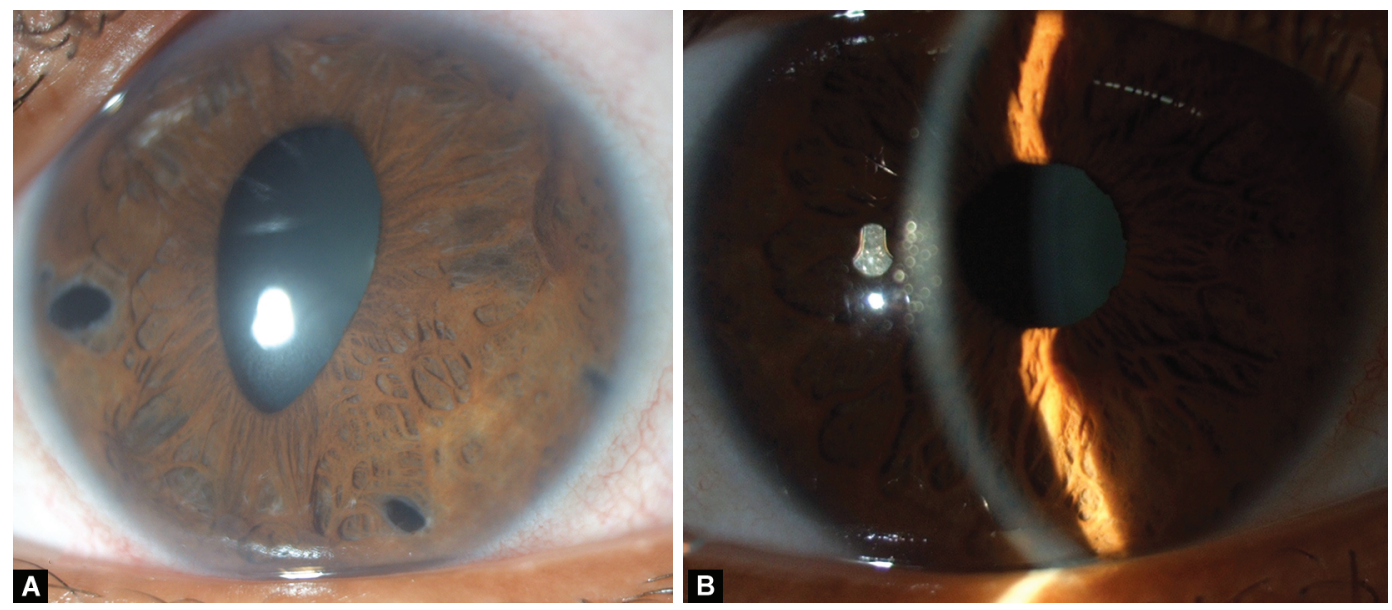

Figs 1 A and B: Slit-lamp photographs of the (A) left eye of the patient (40X) showing clear cornea, multiple iris holes (at 4, 5, 9 o'clock), corectopia, a vertically oval pupil with lost iris pattern. Also, peripheral anterior synechiae (PAS) is present at 2-3 o'clock position; (B) Right eye of the patient $(10 \times)$ showing clear cornea, iris, and anterior segment. The anterior segment is also deep
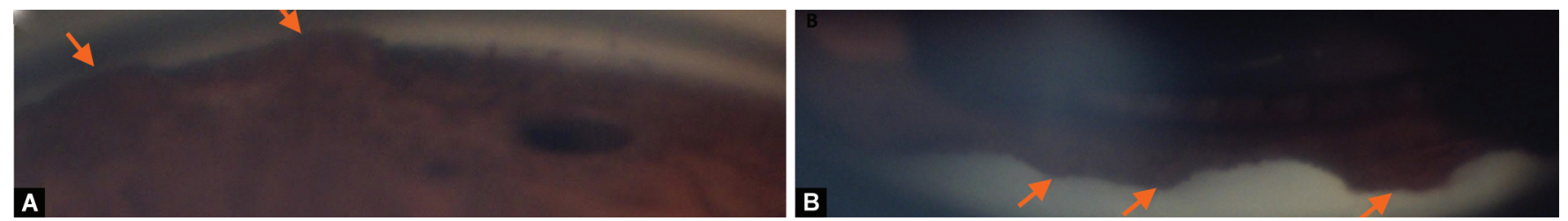

Figs 2A and B: Gonioscopic photographs of (A) superior angle; (B) Inferior angle of the left eye showing closed-angle with scattered peripheral anterior synechiae (orange arrows) throughout the angle
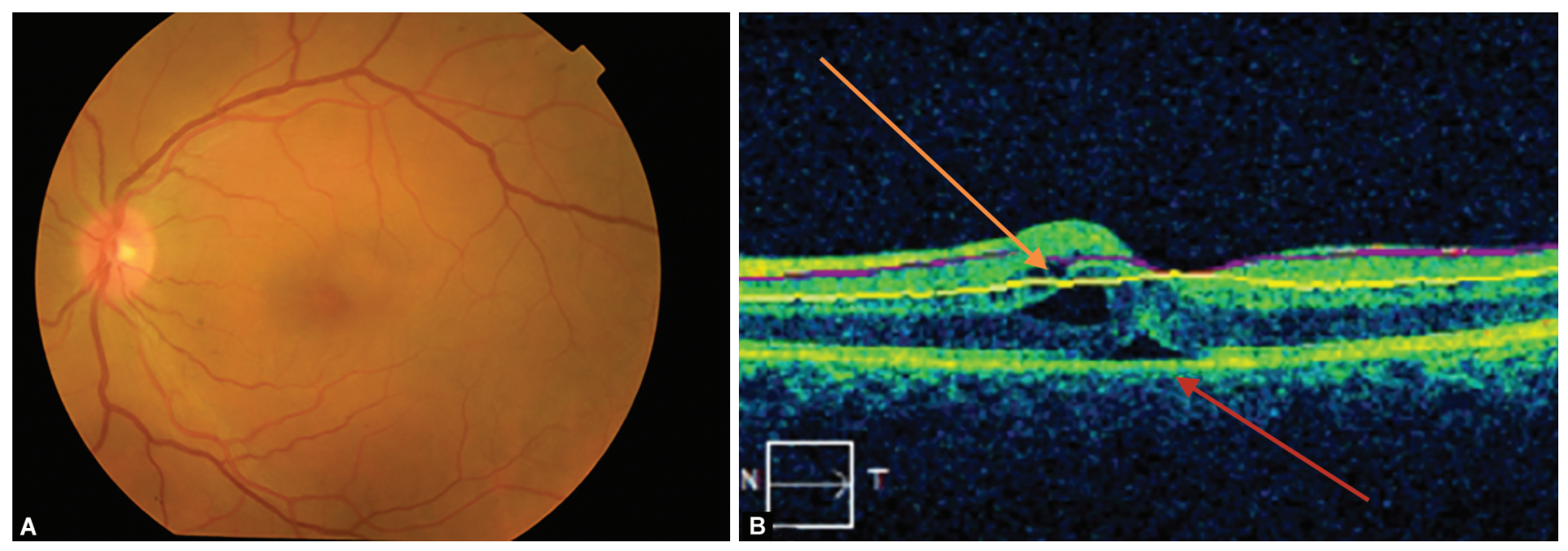

Figs 3A and B: (A) Fundus photograph of the patient showing medium-sized disk with a cup-disk ratio of 0.4:1 with healthy neuroretinal rim and RNFL. Foveal reflex was blunted and about 2-2.5 DD of macular edema was present; (B) OCT macula scan of the left eye showing intraretinal fluid with cystic spaces (macular edema) as shown by the orange arrow with neurosensory detachment (NSD) as shown by the red arrow

of 0.2:1 and 0.4:1 in right and left eye, respectively (Fig. 3A), with a healthy neuroretinal rim and normal macula in both eyes.

Specular microscopy (Table 1) showed significant corneal endothelial cell loss with increased coefficient of variation and decreased hexagonality of cells with characteristic ICE cells in the left eye. Central corneal thickness was 536 and $493 \mathrm{~mm}$ in right and left eye, respectively. Visual fields and OCT-RNFL in both eyes were within normal limits. Based on clinical examination, a diagnosis of ICE syndrome-progressive iris atrophy variant was made in the left eye and she was shifted to a fixed-dose combination of brimonidine with timolol while continuing the other glaucoma medications as were prescribed earlier.

About 2 months later, she presented with a painless, progressive blurring of vision with metamorphopsia in the left eye for the past 1 week. The BCVA decreased in the left eye from $6 / 6$ to $6 / 12$ with stable anterior chamber but on fundus evaluation blunted foveal reflex and about 2-2.5 DD of macular edema was present (Fig. 3A). On the OCT macula of the left eye, intraretinal fluid (macular edema) with NSD was seen (Fig. 3B). She has advised fluorescein angiography but she refused to undergo an invasive investigation. 


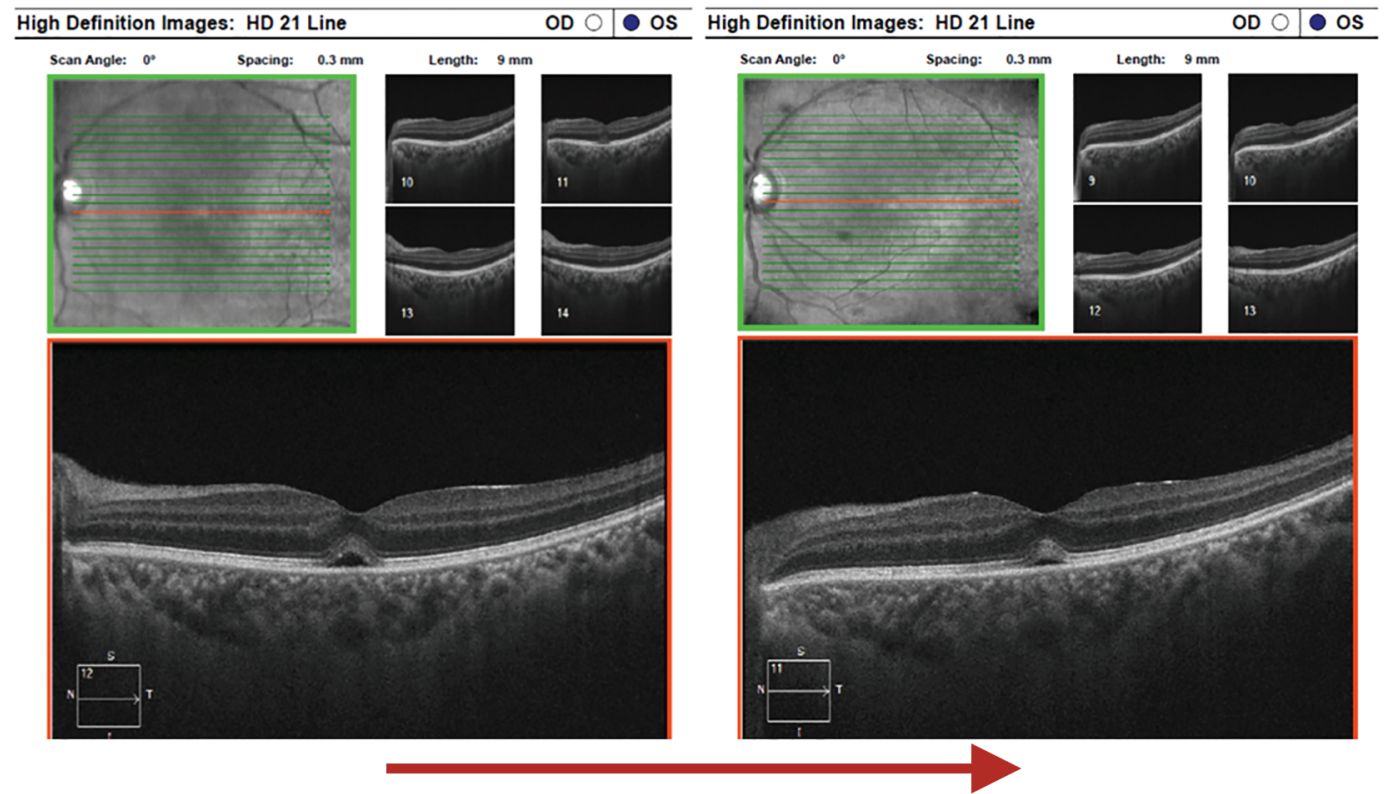

Fig. 4: Serial OCT macula scans of the left scan of the patient showing progressive regression of neurosensory detachment (NSD) over time (from left to right) with treatment

Table 1: Characteristic specular finding of the patient

\begin{tabular}{lcc}
\hline Characteristic finding & Right eye & Left eye \\
\hline Cell density (cells $/ \mathrm{mm}^{2}$ ) & 2,354 & 1,219 \\
Coefficient of variation (CV) & 31 & 57 \\
6A & 53 & 26 \\
Central corneal thickness (CCT) & 536 & 473 \\
\hline
\end{tabular}

She was started on eye drop Nepafenac. After 2 weeks, BCVA in left eye improved to $6 / 9$, GAT-18 $\mathrm{mm} \mathrm{Hg}$, the patient was symptomatically better and macular edema had resolved on fundus evaluation with corroborating findings in OCT but Small NSD was still present (Fig. 4).

In view of unstable IOP even on maximal medical therapy with good compliance, she underwent left eye trabeculectomy with $0.2 \%$ mitomycin on December 14,2018 . The anterior chamber tap for herpes simplex, Epstein-Barr virus, and cytomegalovirus was negative. Histopathological evaluation of iris tissue from iridectomy showed excessive collagen with chronic nonspecific inflammatory cells throughout the stroma. She had an uneventful postoperative course with a good bleb. Neurosensory detachment resolved within 6 weeks of surgery. She has been on regular follow-up for the last 1.5 years postoperatively. Her IOP has been stable in the lower teens. However, she developed clumped iris pigments with the formation of multiple iris nevi at 3,6, 7, and 8 o'clock thus indicating the coexistence of Cogan-Reese syndrome (Fig. 5). There was no structural/functional glaucoma progression without any recurrence of macular edema/NSD.

\section{Discussion}

We present a case of a 36-year-old woman presenting with ICE syndrome who had uncontrolled IOP even on maximum tolerable therapy and developed macular edema along with NSD during the course of management. Macular edema responded well to topical Nepafenac within 2 weeks of treatment, although NSD failed to

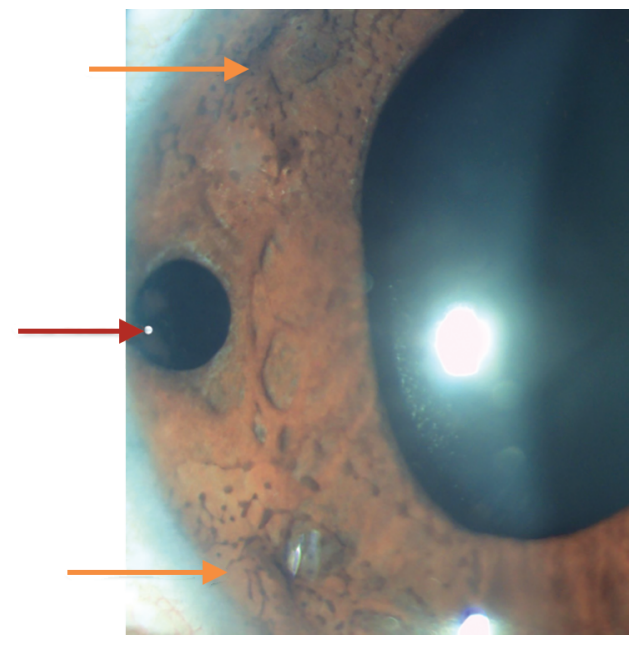

Fig. 5: Slit-lamp photographs of the left eye of the patient (A) 40x; (B) $10 \times$ showing clumped iris pigments with the formation of multiple iris nevi at 3, 6, 7, 8 o'clock (orange arrows) with the iris hole (red arrow) and lost iris pattern indicating coexistence of Cogan-Reese syndrome

respond to it. She was taken up for filtering surgery following which the IOP stabilized and NSD resolved within 6 weeks. Macular edema associated with ICE syndrome has been previously described as a rare association in three case reports. ${ }^{5-7}$ However, our case also highlights the development of NSD with macular edema and ICE syndrome which has not been reported before.

The pathogenesis of macular edema in these cases is unclear. It has been hypothesized that the contraction of abnormal basement membrane formed by proliferation of altered endothelial cells may be responsible for the development of macular edema by disruption of the inner blood-retinal barrier. A viral etiology has also been implicated which causes increased production of prostaglandin-like inflammatory mediators, thus contributing to macular edema..$^{5-7}$ Since topical Nepafenac was effective against macular edema 
in our case, prostaglandin-like material derived from abnormal endothelial cells probably could have caused macular edema although, it might be possible that macular edema would have resolved as a part of the natural history of the disease.

It can also be postulated that prostaglandin (PG) analogs given for the management of glaucoma be a reason for the development of NSD along with macular edema in such cases. They can mimic VEGF and vascular permeability factor (VPF), thus increasing choroidal vascular permeability and impairing the function of the retinal pigment epithelium. Although very few case reports suggest the same and that too no similar cases have been reported with Travoprost $0.004 \% .{ }^{8-10}$ Travoprost has been associated with NSD only in a single case report and the edema resolved on stopping the presumed offending drug. ${ }^{11}$ Macular edema along with NSD has been reported concurrently in only two patients on topical Latanoprost therapy but both of these patients had undergone complicated cataract surgeries and the presence of edema could have been attributed to this risk factor. ${ }^{8,9}$ Since our patient had uncontrolled IOP on maximum therapy, we could not stop eye drop Travoprost despite the rare association of NSD with Travoprost. However, we could discontinue the drop after successful filtering surgery, so we cannot conclusively pinpoint the association of NSD with macular edema with Travoprost in our patient.

Also in prior published case reports, two patients had progressive iris atrophy variant of ICE syndrome whereas one had Cogan-Reese variant..$^{5-7}$ Our patient presented with a progressive iris atrophy variant but simultaneously developed findings of Cogan-Reese over the course of her follow-up. It might be postulated that the coexistence of two variants of ICE syndrome predisposed to the development of macular edema with NSD in our patient. It can also be thought that ICE syndrome along with topical Travoprost therapy predisposed our patient to develop NSD with macular edema.

\section{Conclusion}

It can be suggested that PG analogs are not the good choice for the treatment of secondary glaucoma in patients with ICE syndrome as it can predispose to the development of macular edema with NSD. It might be better to opt for other drugs or even surgical intervention to control IOP in these complicated cases.

\section{References}

1. Campbell DG, Shields MB, Smith TR. The corneal endothelium and the spectrum of essential iris atrophy. Am J Ophthalmol 1978;86(3):317324. DOI: 10.1016/0002-9394(78)90232-5.

2. Wilson MC, Shields MB. A comparison of the clinical variations of the iridocorneal endothelial syndrome. Arch Ophthalmol 1989;107(10):1465-1468.DOI: 10.1001/archopht.1989.01070020539035.

3. Eagle RC, Font RL, Yanoff M, et al. Proliferative endotheliopathy with iris abnormalities. The iridocorneal endothelial syndrome. Arch Ophthalmol 1979;97(11):2104-2111. DOI: 10.1001/archo pht.1979.01020020422002.

4. Aihara M. Glaucoma and iridocorneal endothelial (ICE) syndrome. Ophthalmology 2008;50:1420-1421.

5. Kocaoğlan H, UnlüN, Kanpolat A, et al. Macular edema and iridocorneal endothelial syndrome: a case report. Cornea 2005;24(2):221-223. DOI: 10.1097/01.ico.0000138840.37447.b9.

6. Fourmaux E, Velasque L. Progressive essential iris atrophy associated with chronic cystoid macular edema. J Fr Ophthalmol 2005;28(4):407410. DOI: 10.1016/s0181-5512(05)81073-3.

7. Keita S, Tadashi M, Yui S, et al. Cystoid macular edema associated with iridocorneal endothelial syndrome: a case report. BMC Ophthalmol 2016;16(1):155. DOI: 10.1186/s12886-016-0333-y.

8. Artunay O, Senel A, Sengul A, et al. Central serous chorioretinopathy associated with topical latanoprost therapy. Ocul Immunol Inflamm 2011;19(6):453-455. DOI: 10.3109/09273948.2011.619680.

9. Ozdemir H, Karacorlu M, Karacorlu SA. Serous detachment of macula in cystoid macular edema associated with latanoprost. Eur J Ophthalmol 2008;18(6):1014-1016. DOI: 10.1177/112067210801800627.

10. Besada E, Shechtman D, Frauens BJ. Recurrent serous macular detachment after topical ocular hypotensive medication. Clin Exp Optom 2008;91(6):551-556. DOI: 10.1111/j.1444-0938.2008. 00282.x.

11. Kalikivayi V, Joseph J, Mathews BT, et al. Reversal of retinal pigment epithelial detachment after cessation of topical travoprost therapy. Int Ophthalmol 2018;38(5):2227-2231. DOI: 10.1007/s10792-0170711-3. 